\title{
LA APLICACIÓN DEL DERECHO INTERNACIONAL POR LOS TRIBUNALES nacionales. Reseña del libro de Díaz Fuenzalida, Juan Pablo: Principales tratados internacionales sobre DERECHOS HUMANOS DE LA ONU APLICABLES EN LOS TRIBUNALES de Justicia en Chile. Concordancias con las garantías CONSTITUCIONALES CHILENAS Y JURISPRUDENCIA RELEVANTE DE LOS MÁXIMOS TRIBUNALES. SANTIAGO, Ediciones JuRÍDICAS DE SANTIAGo, 2019, 341 PP.
}

THE APPLICATION Of INTERNATIONAL LAW BY NATIONAL COURTS. REVIEW of the book by Díaz Fuenzalida, Juan Pablo: Principales tratados INTERNACIONALES SOBRE DERECHOS HUMANOS DE LA ONU APLICABLES EN los tribunales de Justicia en CHILE. Concordancias con las garantías CONSTITUCIONALES CHILENAS Y JURISPRUDENCIA RELEVANTE DE LOS MÁXIMOS tribunales. Santiago, Ediciones Jurídicas de Santiago, 2019, 341 PP.

Regina Ingrid Díaz Tolosa* Trabajo recibido el 18 junio de 2019 y aprobado el 19 de junio de 2019

\section{ResUMen}

El sistema universal de protección de los derechos humanos en el ámbito del derecho internacional convencional se compone por nueve tratados que han sido identificados como principales ("The Core International Human Rights Instruments"). Este libro significa un aporte para la implementación de estos en el ordenamiento jurídico chileno, ya que Chile es parte de este sistema y ha ratificado y puesto en vigencia todos ellos, por tanto se encuentra en la obligación de darles cumplimiento de buena fe (principio pacta sunt servanda reconocido en el artículo 26 de la Convención de Viena sobre el Derecho de los Tratados) y todos los órganos del Estado, incluyendo el Poder Judicial, deben adecuar sus actuaciones a lo prescrito en tal plexo normativo internacional.

Palabras clave: recensión, aplicación tratados internacionales, concordancias, jurisprudencia relevante

* Regina Ingrid Díaz Tolosa. Doctora en Derecho, Pontificia Universidad Católica de Chile. Directora de la Carrera de Derecho, Sede Santiago, Universidad Autónoma de Chile. ingrid.diaz@uautonoma.cl 


\begin{abstract}
The universal system for the protection of human rights, with regard to conventional international law, consists of nine treaties that have been identified as main: "The Core International Human Rights Instruments". This book is a contribution for the implementation of these in the Chilean legal system, since Chile is part of this system and has ratified and put into effect all of them, therefore it is in the obligation to give them compliance in good faith (principle pacta sunt servanda recognized in Article 26 of the Vienna Convention on the Law of Treaties) and all State bodies, including the Judiciary, must adapt their actions to the requirements of such international normative plexus.
\end{abstract}

Keywords: review, application of international treaties, concordance, relevant jurisprudence

\title{
1. InTROdUCCIÓN: Fundamentos de La APLICACIÓN DiRECTA DEL DERECHO INTERNACIONAL CONVENCIONAL DE LOS DERECHOS HUMANOS POR LOS TRIBUNALES CHILENOS
}

La obra del académico Juan Pablo Díaz Fuenzalida, titulada "Principales tratados internacionales sobre derechos humanos de la ONU aplicables en los tribunales de justicia en Chile", representa un gran aporte en el ámbito de los Derechos Humanos, pues es una herramienta de utilidad, para estudiantes, abogados y jueces, en cuanto enfatiza las concordancias existentes entre el sistema universal de protección de los derechos humanos, específicamente en relación a los principales tratados que lo componen en el ámbito del derecho internacional convencional, y nuestra Carta Política, junto con destacar jurisprudencia de los tribunales de justicia como ejemplo de aplicación de estas normas internacionales en el orden interno.

Si bien el tema de las relaciones entre el derecho internacional y el derecho interno adolece de un nivel de explicitación tal en nuestra Constitución que impide a la doctrina encontrarse totalmente conteste en todas las aristas que componen el fenómeno ${ }^{1}$, existe un mandato constitucional expreso en el inciso $2^{\circ}$ del artículo $5^{\circ}$ que obliga a todos los órganos del Estado a respetar y promover los derechos esenciales que emanan de la naturaleza humana, sea que estos encuentren su reconocimiento en la propia Constitución o en tratados internacionales ratificados y que se encuentren vigentes ${ }^{2}$. De allí la importancia de identificar estos últimos en el ámbito del sistema universal de derechos humanos y destacar como se complementan con las disposiciones constitucionales que tratan sobre un mismo derecho específico en particular.

A mayor abundamiento, como el propio autor lo destaca en el capítulo introductorio de la obra, esta obligación de dar aplicación al derecho internacional convencional de los derechos humanos no solo se desprende de la disposición constitucional aludida, sino también por el compromiso general del Estado

1 En Chile, desde la inclusión el año 1989 del inciso $2^{\circ}$ del artículo $5^{\circ}$ de la Constitución Política de la República se ha discutido cómo el orden interno incorpora e implementa la normativa internacional, ya que es una cláusula que no se refiere explícitamente, a diferencia de otras constituciones, a relevantes temáticas que orientan las relaciones entre el derecho interno y el internacional, tales como la jerarquía de los tratados, su forma de incorporación, interpretación y aplicación. Vid. v.g. BREWER-CARÍAS (2007) pp. 234-265, quien revisa la situación en Guatemala, Honduras, Colombia, Venezuela, Costa Rica, Perú, Argentina, Panamá, Italia, Alemania, Francia, El Salvador, México, Estados Unidos, Uruguay, República Dominicana, España, Portugal, Chile, Ecuador, Nicaragua, Brasil,

2 Díz (2008), pp. 504-506.

DÍAZ TOLOSA, Regina Ingrid. La aplicación del derecho internacional por los tribunales nacionales. Reseña del libro de Díaz Fuenzalida, Juan Pablo: Principales tratados internacionales sobre derechos humanos de la ONU aplicables en los tribunales de justicia en Chile. Revista Justicia y Derecho, Santiago, v. 2, n 1, 2019 
de Chile para con el derecho internacional convencional adquirido a través de la ratificación en 1981 de la Convención de Viena sobre el Derecho de los Tratados 3 . En efecto, este Convenio ha cristalizado y puesto por escrito derecho consuetudinario, principalmente a través de una serie de principios normativos pertenecientes al derecho internacional general, los cuales precisan las obligaciones de los Estados en el Derecho de los Tratados, entre ellos las disposiciones contenidas en sus artículos 26 y 27, nos permiten deducir la obligación de los tribunales nacionales de dar aplicación a las normas internacionales convencionales.

En efecto, el principio de pacta sunt servanda, el cual de acuerdo con el artículo 26 de la Convención de Viene referida, consiste en que "todo tratado en vigor obliga a las partes y debe ser cumplido por ellas de buena fe", es una norma de origen consuetudinario que constituye un pilar fundamental del Derecho Internacional, considerada una regla de valor objetivo derivada de la idea misma de justicia; ni los acuerdos internacionales ni los derechos internos pueden condicionarlo, respecto a este principio sólo caben dos actitudes, respetarlo o violarlo. Así, el cumplimiento de buena fe de los tratados internacionales implica que los Estados han de actuar de manera consecuente con las obligaciones comprometidas y no pueden desconocerlas, modificarlas o no darles implementación, ya sea omitiéndolas por entero o prefiriendo otras normas que estén en contradicción con ellas o que frustren su propósito. Al contrario, los Estados debieran asegurar la aplicación de estas normas universales, adoptando medidas necesarias para posibilitar su utilización en los órdenes internos, pues si actúan de mala fe, son incongruentes en su actuar y realmente no tienen la intención de obligarse al comprometerse con las normas internacionales preferible sería que no asumieran compromiso internacional alguno.

Por su parte, la imposibilidad de aludir a disposiciones de derecho interno para sustraerse de las obligaciones internacionales asumidas, principio consagrado en el artículo 27 de la Convención de Viena: "una parte no podrá invocar las disposiciones de su derecho interno como justificación del incumplimiento de un tratado", se ha entendido más amplio, en el sentido que no solo impide invocar normas internas contrarias, sino que es deseable armonizar la legislación interna, pues ésta debe servir de complemento a la normativa internacional, y no constituirse en un obstáculo para su aplicación ${ }^{5}$.

Como corolario, los tribunales de justicia como órganos del Estado deben dar aplicación al derecho internacional convencional vigente en el orden interno, y por tanto aún a falta de la deseable armonización legislativa, deberá a través de la interpretación dar una aplicación de los tratados internacionales armoniosa con las normas internas ${ }^{6}$. Entonces, he aquí el provecho patente de esta obra en tanto nos orienta justamente en el camino de la armonización, al señalarnos las concordancias normativas entre constitución y tratado internacional.

En efecto, el libro se estructura en nueve acápites, uno por cada tratado, cada uno de los cuales alude a: 1. Los antecedentes relevantes de cada tratado, tanto a nivel interno como internacional; 2. Las concordancias de la parte dogmática de cada tratado con el artículo 19 de nuestra Carta Fundamental; 3. Juris-

3 Convención de Viena sobre el Derecho de los Tratados. Viena, 23 mayo 1969. Entrada en vigor 27 enero 1980. Ratificado por Chile el 9 de abril de 1981. Promulgada en Chile por el Decreto Supremo n 381 del Ministerio de Relaciones Exteriores, Diario Oficial, 22 junio 1981.

4 Cfr. Heffter (1875) pp. 211 y 490, Wehberg (1959) pp.781-783, Rosenstock (1971) p. 734, Sorensen (1973) pp. 158 y 159, Virally (1983) pp. 130-132, Conforti (1995) p. 17, Benadava (2004) p. 180, Kotzur (2009) pár. 7, 22-25.

5 Cfr. Riveros (1996) pp. 408 y 410, Bustos (1997) p. 76, Llanos (2011) p. 9, Mariño (1999) p. 520, Fuentes (2007) pp. 9 y 10, Nogueira (1996) pp. 348 y 349, Téllez (1998) p. 184, Navarro (2004) pp. 101 y 102, NaSH (2017), pp. 17 y 18.

Troncoso (2012), p. 456, puntualiza que el poder legislativo tiene un "triple desafío: a. desarrollar legislativamente las disposiciones no autoejecutables de los tratados, b. modificar la legislación que sea contraria a los compromisos internacionales del Estado, y c. no dictar leyes que estén en contradicción con dichos compromisos".

6 Cfr. NaSH (2017), pp. 16 y 17.

DÍAZ TOLOSA, Regina Ingrid. La aplicación del derecho internacional por los tribunales nacionales. Reseña del libro de Díaz Fuenzalida, Juan Pablo: Principales tratados internacionales sobre derechos humanos de la ONU aplicables en los tribunales de justicia en Chile. Revista Justicia y Derecho, Santiago, v. 2, n 1, 2019 
prudencia chilena seleccionada como ejemplo de aplicación de estos tratados en diversas materias, tales como derecho civil, penal, tributario ${ }^{7}$ y laboral, entre otros; 4. Consideraciones finales a modo de conclusión. Así, se trata de un texto con un estilo lúdico que permite al lector la consulta parcial de su contenido de acuerdo con la necesidad que se le presente. Además, incorpora cuadros resúmenes de los antecedentes internacionales y nacionales de cada tratado, siendo muy ameno y ordenado de seguir para el lector.

\section{ConCordancias ENTRE LOS ÓRDENES INTERNACIONAL Y NACIONAL EN MATERIA DE DERECHOS HUMANOS}

Chile es Estado miembro de la ONU desde el 24 de octubre de 1945. Si bien es cierto que el objetivo principal de la ONU es el mantenimiento de la paz y seguridad internacionales, la Carta de las Naciones Unidas establece para los Estados miembros la obligación de un compromiso real y obligatorio para con la promoción y protección de los derechos humanos,

a través de sus artículos 55 y 56, al disponer que "todos los miembros se comprometen a realizar acciones conjuntas o por separado, en cooperación con la Organización", para alcanzar el propósito "de respeto universal y observancia de los derechos humanos y libertades fundamentales de todos, sin distinción de raza, sexo, idioma o religión". Así, los Estados firmantes de la Carta han reconocido que los derechos humanos son un tema de interés internacional y dejan de pertenecer exclusivamente a las jurisdicciones nacionales; el sistema universal de protección de los derechos humanos es coadyuvante y complementario del derecho interno de los Estados ${ }^{8}$. Aunque en los primeros años de vigencia de la Carta de la ONU se discutió si ella imponía realmente obligaciones a los Estados en materia de derechos humanos, hoy se entiende que los Estados se han comprometido a cooperar en el plano internacional y, también, a adoptar medidas en el plano interno para el respeto y protección de los derechos humanos. Así, el principio general de respetar los derechos humanos, y por ende la dignidad intrínseca de la persona humana (por el sólo hecho de ser persona) deriva su más sólida garantía en el Sistema de las Naciones Unidas.

Chile en un Estado democrático en el cual unos de los pilares fundamentales es el respeto de la persona y su dignidad, por lo que existe confianza en que la institucionalidad y sus representantes, utilizarán todos los medios disponibles para otorgar una efectiva garantía a sus derechos, entre estos mecanismos, ciertamente debemos incluir al Derecho Internacional de los Derechos Humanos ${ }^{10}$. Los instrumentos internacionales de carácter universal, adoptados por las Naciones Unidas, muestran la existencia de un consenso internacional sobre estándares mínimos de protección de los derechos humanos, por tanto, las normas internas e internacionales no se desplazan ni se trasponen, sino que se complementan en beneficio de los individuos. Además, se destaca que la Carta alude expresamente al principio pacta sunt servanda, al establecer en su artículo 2.2 que "[I]os Miembros de la Organización, a fin de asegurarse los derechos y beneficios inherentes a su condición de tales, cumplirán de buena fe las obligaciones contraídas por ellos de conformidad con esta Carta".

7 Díaz (2019), pp. 74-76, destaca voto disidente de Ministro excelentísimo, señor Künsemüller, pues "trae a colación dicho caso porque se utiliza incluso en áreas que en principio no se vienen a la mente en asuntos de derechos humanos, cual es, los derechos fundamentales en materia tributaria, en que el Ministro intenta aplicar el tratado en resguardando derechos del contribuyente, en concreto a ser juzgado en un plazo razonable, lo que acarrearía la prescripción de la obligación y/o acción de cobro del tributo".

8 Pastor (1991) p. 202; Brewer-Carías (2007), pp. 231 y 232; Albár (2008) pp. 34-36; NASH (2017), pp. 18 y 19.

9 FenWick (1963) pp. 47, 203 y 204, CASSESe (2005) p. 59, SHAw (2008) pp. 276 y 277.

10 Schachter (1983) p. 849, Nogueira (1996) p. 351, Nogueira (2003) p. 405, Complak (2005) p. 25, De Miguel (2005) pp. 334 y 335 , Mariño (2005) p. 831, Nogueira (2008) pp. 265 y 266, Aldunate (2008) pp. 98 y 99, Viteri (2012) pp. 119-122.

DÍAZ TOLOSA, Regina Ingrid. La aplicación del derecho internacional por los tribunales nacionales. Reseña del libro de Díaz Fuenzalida, Juan Pablo: Principales tratados internacionales sobre derechos humanos de la ONU aplicables en los tribunales de justicia en Chile. Revista Justicia y Derecho, Santiago, v. 2, n 1, 2019 
Luego, los primeros Ilamados a su aplicación son los operadores jurídicos del orden interno, pues recordemos que la jurisdicción internacional es de carácter excepcional y subsidiario ${ }^{11}$. Además, en el ámbito universal se carece de un Tribunal de Derechos Humanos, aunque las comisiones y comités que se han creado para supervigilar el cumplimiento de estos tratados internacionales universales ejercen funciones de control y contienen fórmulas de trabajo bajo el principio de contradictorio, no son órganos judiciales ni tienen capacidad para adoptar resoluciones calificables de sentencias ${ }^{12}$. Por otra parte, sólo los informes periódicos operan de forma automática, pues los órganos internacionales de control no pueden recibir denuncias sin que el Estado denunciado haya aceptado previamente tal posibilidad ${ }^{13}$.

Así las cosas, se ha de considerar que debido a la carencia de una jurisdicción internacional de derechos humanos suprema de cierre obligatorio y la inexistencia de normas que establezcan y regulen procedimientos autónomos de ejercicio obligatorio para hacer cumplir coercitivamente las normas de orden público internacional, particularmente en caso de violaciones graves, es importante que el Estado sea consciente de que es parte de un sistema internacional de protección de los derechos de las personas, y que actúe en consecuencia, de manera tal de suplir en el ordenamiento interno las carencias funcionales del orden internacional, movidos por un espíritu de cooperación multilateral ${ }^{14}$.

La obra en comento contribuirá a fomentar esta cooperación y el cumplimiento del deber de respeto de los derechos humanos en Chile, ya que se refiere a las concordancias existentes entre nuestra Constitución y los principales tratados sobre derechos humanos del sistema universal. Estos son nueve y han sido reconocidos como el núcleo del sistema. Los Pactos de 1996, sobre derechos civiles y políticos y sobre derechos económicos, sociales y culturales, son instrumentos generales, en cuanto contemplan al individuo de forma genérica (no a un grupo o categoría determinados de personas) y a los derechos humanos en su globalidad (varios derechos circunscritos en las categorías de civiles, políticos, económicos, sociales y culturales), pero la ONU también ha ido codificando la protección de los derechos humanos en instrumentos específicos, ya sea porque se dirigen a la protección de algún grupo determinado de individuos (mujeres, niños, extranjeros, personas con discapacidad), sea porque desarrollan de manera exclusiva la erradicación de determinada conducta que agravia desmesuradamente la dignidad humana (discriminación racial, tortura y otros tratos o penas crueles, inhumanos o degradantes, desapariciones forzadas de personas). Esta normativa más específica o particular, en los términos explicados, refuerza o profundiza el sistema general constituido por los Pactos ${ }^{15}$.

El Estado de Chile, a través de la ratificación y puesta en vigencia de todos estos tratados, ha reconocido la existencia de un orden internacional mínimo, cuyos principios básicos que lo sostienen son la paz y seguridad internacionales y el respeto de la dignidad humana. Y, por tanto, el ser el Estado miembro activo de la comunidad internacional implica el respeto de ciertas normas, si no fuera así la sociedad se mantendría en una condición de salvajismo descrita por la frase homo homini lupus, la cual duró hasta las guerras mundiales ${ }^{16}$.

11 GonzÁlez (2017), p. 719, sobre la base del principio de subsidiariedad, "sostiene que la libertad que goza un Estado para determinar el alcance de los derechos humanos a nivel nacional depende fundamentalmente del nivel de protección a la dignidad humana reconocido por el derecho de los tratados".

12 Revisar en la obra que se comenta los cuadros con antecedentes internacionales del tratado, en cada uno de ellos se específica cuál es el organismo encargado de su supervisión. Asimismo, normalmente en la parte no dogmática del tratado se especifica la regulación de estos.

13 Medina y Nash (2003) pp. 43-51, Diez de Velasco (2007) p. 651, Schachter (1994) pp. 17 y 18, Benadava (2004) pp. 210-215, Cassese (2005) p. 320, Albár (2008) p. 33, Aldunate (2008) p. 35, Shaw (2008) pp. 303-311, Oficina del Alto Comisionado para los Derechos HumaNOS DE LAS NACIONES UNIDAS (2012), pp. 22-42.

14 Cfr. v.g. Mariño (2005) pp. 827 y 828, Diez de Velasco (2007) p. 651, Carrillo-Santarelli (2012) pp. 839 y 840, Iglesias (2013), p. 167.

15 Cfr. Medina y NASH (2003) p. 44, SHaw (2008) pp. 280-302, Iglesias (2013), p. 170.

16 Cfr. Kelsen (1965) pp. 131 y 132, Jiménez de AreChaga et al. (2005) p. 367, Lepard (2010) p. 244.

DÍAZ TOLOSA, Regina Ingrid. La aplicación del derecho internacional por los tribunales nacionales. Reseña del libro de Díaz Fuenzalida, Juan Pablo: Principales tratados internacionales sobre derechos humanos de la ONU aplicables en los tribunales de justicia en Chile. Revista Justicia y Derecho, Santiago, v. 2, n 1, 2019 
El libro, entonces nos conduce a través del tiempo, ya que los tratados se presentan ordenados de conformidad a la fecha de su adopción internacional, indicándonos en cada caso su estado de ratificación y puesta en vigencia por Chile, para luego indicarnos por cada uno de los artículos de la parte dogmática del tratado, su correspondencia con las diversas garantías constitucionales reconocidas en el texto constitucional vigente.

\section{JURISPRUDENCIA RELEVANTE, EJEMPLO DE APLICACIÓN INTERNA DEL DERECHO INTERNACIONAL DE LOS DERECHOS HUMANOS}

En acápites anteriores ya hemos destacado que los órganos jurisdiccionales internos cumplen una relevante función en el control de la interpretación y aplicación de las normas internacionales en el orden estatal, desde el momento en que tales reglas jurídicas pueden fijar los derechos y obligaciones de los particulares en los procedimientos judiciales internos ${ }^{17}$. Este papel ha sido reconocido por nuestros propios tribunales, quienes no han desconocido la existencia del sistema universal de protección de los derechos humanos, todo lo contrario, en ocasiones para fundamentar la obligación de respeto de estos derechos en sus sentencias alude expresamente a normas internacionales específicas ${ }^{18}$, o bien, con una cláusula más general, con alusión a los paradigmas generales del Derecho Internacional de los Derechos Humanos consagrados en tratados internacionales ratificados por Chile ${ }^{19}$.

Así es constatado en esta obra, la cual en el apartado referente a "Jurisprudencia sobre aplicación del tratado", siguiendo un estilo didáctico, incluye un cuadro con la identificación de la sentencia y un resumen del caso, para luego mostrarnos extracto con consideraciones textuales realizadas en la sentencia. Consecuentemente es posible observar que los jueces estatales contribuyen a moldear el Derecho internacional a través de su aplicación directa interna ${ }^{20}$, además de efectuar una indirecta al interpretar su legislación doméstica a la luz de las normas internacionales ${ }^{21}$, así por ejemplo, en la sentencia del Tribunal Constitucional de 2012 que considera la Convención Internacional sobre la Eliminación de todas las Formas de Discriminación Racial en la creación de un concepto jurisprudencial de discriminación arbitraria22, y la del 2014 que considera la Convención Internacional sobre la Protección de los Derechos de todos los Trabajadores Migratorios y de sus Familiares para determinar la conceptualización del elemento normativo del tipo penal "esclavitud o prácticas análogas a ésta"23.

17 IGLESIAS (2013), pp. 165 y 166.

18 Así, v.g., Díaz (2019), pp. 93-97, respecto a la aplicación directa del artículo 13 del Pacto Internacional de Derechos Económicos, Sociales y Culturales; Díaz (2019), pp. 118-121, respecto a la aplicación directa del artículo 11 de la Convención sobre la eliminación de todas las formas de discriminación contra la mujer; Díaz (2019), pp. 184-187, respecto a la aplicación directa del artículo 3 de la Convención sobre Derechos del Niño; Díaz (2019), pp. 291-295, respecto a la aplicación directa del artículo 2 de la Convención sobre los derechos de las personas con discapacidad.

19 Cfr. v.g. Díaz (2019), p. 148, considerando 9i Díaz (2019), p. 327, considerando $1^{\circ}$.

20 Vid. nota supra 18.

21 Cfr. NASH (2012), pp. 46-50, IGLESIAS (2013), pp. 168 y 169.

22 Díaz (2019), pp. 39-43.

23 Díaz (2019), pp. 243-248.

DÍAZ TOLOSA, Regina Ingrid. La aplicación del derecho internacional por los tribunales nacionales. Reseña del libro de Díaz Fuenzalida, Juan Pablo: Principales tratados internacionales sobre derechos humanos de la ONU aplicables en los tribunales de justicia en Chile. Revista Justicia y Derecho, Santiago, v. 2, n 1, 2019 


\section{UTILIDAD O USOS DE UN LIBRO DE CONCORDANCIAS NORMATIVAS Y JURISPRUDENCIA RELEVANTE}

Por tanto, habida consideración de los comentarios más arriba desarrollados, sin lugar a duda, este libro proporcionará una herramienta de utilidad en el estudio y práctica de las relaciones entre el derecho interno y el internacional, ya que el profesor Díaz Fuenzalida contribuye con esta obra a la orientación de los operadores jurídicos en tanto respecto de cada uno de los nueve tratados revisa sus concordancias con la Carta Magna para luego destacar jurisprudencia de nuestros tribunales en los cuales se ha hecho alusión e implementación del tratado para la resolución de casos concretos.

De esta forma, muestra que las concordancias entre los tratados y la Constitución son un ejercicio necesario en tanto la dignidad de la persona humana es un bien jurídico protegido en común por los sistemas nacional e internacional, y por tanto la interpretación de sus textos, para determinar el contenido de cada uno de los derechos protegidos, debe ser armónica, sistemática y finalista, en el sentido de buscar la fórmula que mejor proteja al individuo (principio pro homine o de la progresividad en la interpretación de los derechos humanos ${ }^{24}$. Luego, una protección efectiva de los derechos humanos a través de la aplicación de tratados universales de Derecho Internacional de los Derechos Humanos por la judicatura nacional es posible.

\section{Bibliografía Citada}

Albár Álvarez, Juan Pablo (2008): El Derecho Internacional de los Derechos Humanos y el Derecho Internacional Humanitario (Santiago, Legal Publishing).

Aldunate Lizana, Eduardo (2008): Derechos Fundamentales (Santiago, Legal Publishing).

Benadava, Santiago (2004): Derecho Internacional Público (8ª edición, Santiago, Abeledo Perrot).

BREWER-CARÍAs, Allan R. (2007): "La aplicación de los tratados internacionales sobre derechos humanos en el orden interno de los países de América Latina", Revista IIDH, Vol. 46: pp. 219-271.

Bustos ValderRama, Crisólogo (1997): "La incorporación de los tratados en el derecho interno chileno. Análisis desde la perspectiva del Derecho internacional", lus et Praxis (vol. 2 n²): pp. 73 -80.

Carrillo-Santarelli, Nicolás (2012): "Enhanced multi-level protection of human dignity in a globalized context through humanitarian global legal goods", German Law Journal (vol 13 n 7): pp. $829-873$.

CASSESE, Antonio (2005): International Law (2 ${ }^{\text {nd }}$ edition, Oxford, Oxford University Press).

Complak, Krystian (2005): "Por una comprensión adecuada de la dignidad humana", Díkaion (vol. 19 n 14): pp. 19 - 30.

ConfortI, Benedetto (1995): Derecho Internacional (Traducc. Raúl Vinuesa, Buenos Aires, Zavalia).

De Miguel Beriain, Iñigo (2005): "La dignidad humana, fundamento del derecho", BFD: Boletín de la Facultad de Derecho (n²7): pp. $325-356$.

24 BREWER-CARIAS (2007), p. 270 interpreta que este principio está incorporado implícitamente en nuestra Carta Magna, pues precisa como fin esencial del Estado el respeto de los derechos humanos en el artículo $5^{\circ}$ : "es "deber de los órganos del Estado respetar y promover tales derechos, garantizados por esta Constitución, así como por los tratados internacionales ratificados por Chile y que se encuentren vigentes".

DÍAZ TOLOSA, Regina Ingrid. La aplicación del derecho internacional por los tribunales nacionales. Reseña del libro de Díaz Fuenzalida, Juan Pablo: Principales tratados internacionales sobre derechos humanos de la ONU aplicables en los tribunales de justicia en Chile. Revista Justicia y Derecho, Santiago, v. 2, n 1, 2019 
Diez de Velasco, Manuel (2007): Instituciones de Derecho Internacional Público (16a edición, Madrid, Editorial Tecnos).

Díaz Fuenzalida, Juan Pablo (2019): Principales tratados internacionales sobre derechos humanos de la ONU aplicables en los tribunales de justicia en Chile. Concordancias con las garantías constitucionales chilenas y jurisprudencia relevante de los máximos tribunales (Santiago, Ediciones Jurídicas de Santiago).

Díaz Tolosa, Regina Ingrid (2008): "Pautas de interpretación de los tratados internacionales derivadas del deber de promoción de los derechos humanos impuesto a la judicatura chilena por la Constitución Política", Anuario de Derecho Constitucional Latinoamericano 2008: pp. 499-522.

Fenwick, Charles G. (1963): Derecho Internacional (Traducc. María Eugenia I. de Fischman de la 3a edición en inglés, 1952, Buenos Aires, Omeba).

Fuentes Torrijo, Ximena (2007): "El derecho internacional y el derecho interno: definitivamente una pareja dispareja", Revista de Economía y Derecho, Vol. 15, № 4: pp. 2-35.

González Domínguez, Pablo (2017): "Reconfiguración de la relación entre el derecho internacional de los derechos humanos y el derecho nacional sobre la base del principio de subsidiariedad", Anuario Mexicano de Derecho Internacional, №. 17: pp. 719-750.

Heffter, Agosto Wilhelm (1875): Derecho internacional Público de Europa (Traducc. G. Lizarraga, Madrid, Librería de Victoriano Suarez).

IGLESIAS VeLAsCo, Alfonso J. (2013): "Reflexiones sobre la implementación de los tratados internacionales por los tribunales domésticos: especial referencia a España", Anuario Español de Derecho Internacional, vol. 29: pp. 165-216.

Jiménez de Aréchaga, Eduardo, Arbuet-Vignal, Heber y Pucelro Ripoll (2005): Roberto, I Derecho internacional Público. Principios, normas y estructuras (Montevideo, Fundación de Cultura Universitaria).

Kelsen, Hans (1965): Principios de Derecho Internacional Público (Traducc. Hugo Caminos y Ernesto Hermida, Buenos Aires, El Ateneo).

Kotzur, Markjus (2009): "Good Faith (bona fide)", Max Planck Encyclopedia of Public International Law, [fecha consulta: 19junio 2019]. Disponible en <https://opil.ouplaw.com/view/10.1093/law:epil/9780199231690/ law-9780199231690-e1412?rskey=m2yYrb\&result=5\&prd=OPIL>.

LEPARD, Brian D. (2010): Customary International Law. A new theory with practical requirements (New York, Cambridge University Press).

Llanos Mansilla, Hugo (2011): IV Teoría y Práctica del Derecho internacional Público. Las relaciones entre el Derecho internacional y el derecho interno (Santiago, Editorial Jurídica de Chile) 450 pp.

Mariño Menéndez, Fernando (1999): Derecho Internacional Público. Parte General (Madrid, Editorial Trotta).

Medina Quiroga, Cecilia y Nash Rojas, Claudio (2003): "Parte I. Doctrina. A. El sistema internacional de protección de los derechos humanos y B. Importancia y alcance de los instrumentos internacionales de derechos humanos en el ámbito nacional", Defensoría Penal Pública, Manual de Derecho internacional de los derechos humanos para defensores penales públicos (Santiago, Centro de Documentación Defensoría Penal Pública) pp. 18 - 84.

NaSH Rojas, Claudio (2012): Derecho Internacional de los Derechos Humanos en Chile. Recepción y aplicación en el ámbito interno (Santiago, Centro de Derechos Humanos, Universidad de Chile).

(2017): "Los usos del Derecho Internacional de los Derechos Humanos en la jurisprudencia de los

DÍAZ TOLOSA, Regina Ingrid. La aplicación del derecho internacional por los tribunales nacionales. Reseña del libro de Díaz Fuenzalida, Juan Pablo: Principales tratados internacionales sobre derechos humanos de la ONU aplicables en los tribunales de justicia en Chile. Revista Justicia y Derecho, Santiago, v. 2, n 1, 2019 
Tribunales Superiores de Justicia en Chile", Estudios Constitucionales, Año 15, № 1, pp. 15-54.

Navarro Dolmestch, Roberto (2004): "Los efectos en el sistema chileno de fuentes del Derecho Penal de la incorporación de los tratados internacionales y del fenómeno de la globalización", Ius et Praxis (vol. 10 n 1): pp. $77-111$.

Nogueira AlcalÁ, Humberto (1996): "Los tratados internacionales en el ordenamiento jurídico chileno", Revista Chilena de Derecho (vol. 23 n 2 y 3, tomo I): pp. 341 - 380.

(2003): "Los derechos esenciales o humanos contenidos en los tratados internacionales y su ubicación en el ordenamiento jurídico nacional: doctrina y jurisprudencia", lus et Praxis (vol. 9 n 1): pp.403 $-466$.

(2008): "El boque constitucional de derechos: La confluencia del derecho internacional y del derecho constitucional en el aseguramiento y garantía de los derechos fundamentales en América Latina", Llanos Mansilla, Hugo y Picand AlbónICO, Eduardo (coordinadores académicos), I Derecho internacional Público. Estudios de Derecho internacional. Libro Homenaje al profesor Santiago Benadava (Santiago, Editorial Librotecnia) pp. 265 - 304.

Oficina del Alto Comisionado para los Derechos Humanos de las Naciones Unidas (2012): El sistema de tratados de derechos humanos de las Naciones Unidas. Folleto informativo No 30/Rev.1 (New York y Ginebra, Naciones Unidas).

PAstor RIDRUejo, José Antonio (1991): Curso de Derecho internacional público y organizaciones internacionales (Reimpresión 3a edición, Madrid, Tecnos).

Riveros Marín, Edgardo (1996): "Las relaciones entre el derecho internacional y el derecho interno. Notas sobre el caso chileno", Revista Chilena de Derecho (vol. 23 ns 2 y 3, Tomo I): pp. 407 - 410.

Rosenstock, Robert (1971): "The Declaration of Principles of International Law Concerning Friendly Relations: A Survey", American Journal of International Law (vol. 65 n 5): pp. 713 - 735.

SCHACHTER, Oscar (1983): "Human dignity as a normative concept", American Journal of International Law (vol. $\left.77 n^{\circ} 4\right)$ : pp. $848-854$.

SHAW, Malcolm N. (2008): International Law (6 $6^{\text {th }}$ edition, Cambridge, Cambridge University Press).

Sorensen, Max (ed.) (1973): Manual de Derecho Internacional Público (Traducc. a cargo de la Dotación Carnegie para la Paz Internacional, México D.F., Fondo de Cultura Económica).

Téllez Soto, Claudia (1998): "Valor jurídico de los tratados internacionales en el derecho interno", Revista de Derecho Universidad Austral (vol. 9): pp. 179 - 190.

Troncoso Repetto, Claudio (2012): "Derecho internacional, constitución y derecho interno”, Revista de Derecho Público, vol. 77: pp. 453-462.

VIRALly, Michel (1983): "Review Essay: Good Faith in Public International Law", American Journal of International Law (vol. 77 n 1): pp. 130 - 134.

VITERI Custodio, Daniela Damaris (2012): "La naturaleza jurídica de la dignidad humana: un análisis comparado de la jurisprudencia del Tribunal Constitucional español y el Tribunal Constitucional Federal alemán", Estudios de Derecho (Universidad de Antioquia) (vol. 69 n 153): pp. 113 - 145.

WeHBERG, Hans (1959): "Pacta sunt servanda", American Journal of International Law (vol. 53 n 4): pp. 775 786.

DÍAZ TOLOSA, Regina Ingrid. La aplicación del derecho internacional por los tribunales nacionales. Reseña del libro de Díaz Fuenzalida, Juan Pablo: Principales tratados internacionales sobre derechos humanos de la ONU aplicables en los tribunales de justicia en Chile. Revista Justicia y Derecho, Santiago, v. 2, n 1, 2019 